\title{
Efecto de la suplementación de grasas sobre características productivas, tasas de preñez y algunos metabolitos de los lípidos en vacas para carne en pastoreo
}

\author{
Effect of fat supplementation on productive traits, pregnancy rates and some \\ lipid metabolites in beef cows on range \\ JL Espinoza-Villavicencio ${ }^{a^{*}}$, R Ortega-Pérez ${ }^{\mathbf{b}}$, A Palacios-Espinosa ${ }^{a}$, A Guillén-Trujillo ${ }^{a}$ \\ aDepartamento de Zootecnia, Universidad Autónoma de Baja California Sur, La Paz, B.C.S., México. \\ ${ }^{\text {b} C e n t r o ~ d e ~ I n v e s t i g a c i o n e s ~ B i o l o ́ g i c a s ~ d e l ~ N o r o e s t e ~(C I B N O R), ~ L a ~ P a z, ~ B . C . S ., ~ M e ́ x i c o . ~}$
}

\begin{abstract}
SUMMARY
In order to evaluate the effect of a supplementation with two sources of fat in beef cows on range, 120 adult females in two herds were used. One herd of 60 Charolais cows (A) and another herd of 60 Chinampo cows (B, local Creole) were fed $(2 \mathrm{~kg} / \mathrm{cow} /$ day) isoproteic concentrates $(\mathrm{PC}=16.6 \%)$ with a similar content of ME (aprox. $3.0 \mathrm{Mcal} / \mathrm{kg}$ ) during a 90 days period (initiating 30 days precalving), respectively. In each herd, cows were randomly assigned into three groups. The control groups $(\mathrm{TE} ; \mathrm{n}=20$ ) were fed a supplement containing $3.2 \%$ of fat. Another group of 20 cows per herd was fed a supplement containing additional fat $(13.2 \%)$ as calcium soaps of fatty acids (JCAG). The third group (SB) within each herd $(\mathrm{n}=20)$ was fed a supplement containing $13.4 \%$ of fat (bovine tallow). Orthogonal contrasts (TE vs SB + JCAG and SB vs JCAG) were used to compare the means of non categorical variables. In herd A, milk yield was greater at $35(\mathrm{P}<0.05)$ and $90(\mathrm{P}<0.01)$ days of lactation in cows fed supplemental fat $(\mathrm{SB}+\mathrm{JCAG})$ than TE treatment. In both herds, weight of calves at 35 and 90 days of age, body weight and body condition score of cows at 90 days postcalving were higher in SB + JCAG than TE $(\mathrm{P}<0.01)$. Serum cholesterol, high density lipoproteins, low density lipoproteins and triglycerides were greater in cows supplemented with fat $(\mathrm{SB}+\mathrm{JCAG})$ than TE treatment $(\mathrm{P}<0.01)$. Pregnancy rates in both herds were higher in fat supplemented cows $(\mathrm{P}<0.05)$. We concluded that additional fat incorporated in a range supplement during pre- and postpartum periods increased milk yield, growth of calves, body weight and condition score of cows, lipid metabolites and pregnancy rates.
\end{abstract}

Palabras clave: vacas para carne, pastoreo, suplementación, grasa.

Key words: beef cows, range, supplementation, fat.

\section{INTRODUCCIÓN}

En las regiones desérticas del noroeste de México el ganado bovino para carne se mantiene gran parte del año en áreas de pastoreo basadas en pastos y arbustos en estado fenológico de latencia, forrajes que durante esa etapa tienen una calidad nutricional menor a las demandas de los animales para llenar sus requerimientos (Gutiérrez 1991). La limitación de ciertos nutrientes como la energía, el nitrógeno disponible en el rumen y los aminoácidos contribuyen a una productividad animal inferior a lo deseado (Titgemeyer y Loëst 2001).

Es sabido que la nutrición tiene efectos importantes en la eficiencia reproductiva (Hess y col 2005). Tanto las tasas de preñez como la actividad ovárica posparto son afectadas en vacas que han tenido un consumo restringido de energía antes y/o después del parto y para que una vaca continúe en un ciclo de producción anual debe de preñarse dentro de los 90 días que siguen al parto (Freetly y col 2006).

\footnotetext{
Aceptado: 08.07.2009.

* Nicolás Bravo \# 419, entre Guillermo Prieto y Serdán, Colonia
} Centro, CP 23000, La Paz, B.C.S., México; jlvilla@uabcs.mx
La suplementación de lípidos en la dieta ha sido utilizada para cubrir las demandas nutricionales asociadas a la producción de leche y reproducción (Hess y col 2005), observándose además que las grasas pueden tener efectos positivos directos sobre la reproducción en el ganado bovino para carne (Funston 2004) incrementando la fertilidad (Espinoza y col 1995). Se han estudiado varias fuentes de ácidos grasos en la dieta de rumiantes como la semilla de girasol, semilla de cártamo, semilla de algodón, salvado de arroz, pasta de soya, harina de pescado, sebo animal y las sales de calcio de ácidos grasos, observándose que su consumo influye en algunas funciones reproductivas (Funston 2004).

Las grasas de la dieta influyen en la reproducción alterando el tamaño del folículo preovulatorio (Zachut y col 2008), reduciendo el intervalo a la primera ovulación posparto en bovinos de carne, incrementando la concentración de progesterona durante la fase lútea del ciclo estral, modulando la síntesis de prostaglandina en el útero y mejorando la calidad y capacidad de desarrollo tanto del ovocito y del embrión (Santos y col 2008).

Se ha especulado que los lípidos de la dieta actúan como agentes distribuidores de nutrientes que pueden cambiar el uso de la energía de un proceso metabólico a otro, incrementando con ello el potencial de las vacas para 
almacenar grasa corporal y ganar o mantener su condición corporal (Bottger y col 2002). La incorporación de grasas protegidas de la digestión ruminal (Moallem y col 2007) o de sebo de bovino (Bobe y col 2009) a la dieta de vacas lecheras incrementa la producción de leche. Sin embargo, en bovinos para carne ese tipo de estudios son escasos, a pesar de que la producción de leche es uno de los factores que determinan el peso de las crías al destete (Auchtung y col 2002).

De acuerdo a los planteamientos anteriores, la suplementación de grasas a vacas para carne explotadas en sistemas de producción de pastoreo en agostadero podría servir como una estrategia para mejorar el comportamiento productivo y reproductivo. Es por eso que el objetivo del presente trabajo fue evaluar el efecto de la grasa bovina y de una grasa protegida de la digestión en el rumen sobre la producción de leche, crecimiento de los terneros, peso y condición corporal, concentración sérica de algunos metabolitos de los lípidos y tasas de preñez de vacas para carne en pastoreo.

\section{MATERIAL Y MÉTODOS}

\section{DESCRIPCIÓN DEL ÁREA DE ESTUDIO}

El presente trabajo se realizó en dos hatos del municipio de La Paz, Baja California Sur, México, uno ubicado en las coordenadas geográficas $23^{\circ} 62^{\prime} \mathrm{N}$ y $110^{\circ} 18^{\prime} \mathrm{O}$, y el otro en $23^{\circ} 44^{\prime} \mathrm{N}$ y $110^{\circ} 11^{\prime} \mathrm{O}$, ambos a 300 metros sobre el nivel del mar. El clima predominante es muy seco en sus variantes semicálido y cálido, con lluvias en verano, principalmente y ocasionales en el invierno. La precipitación media anual es de $300 \mathrm{~mm}$ con una temperatura media anual de $22,07^{\circ} \mathrm{C}$ (DGTENAL 1980).

Las especies vegetales de las áreas de pastoreo a las cuales tuvieron libre acceso los animales de ambos hatos es matorral sarcocaulescente y la flora constituida principalmente por especies propias de zonas desérticas como Bursera microphylla, Cyrtocarpa edulis, Fouquieria diguetii, Jatropha cinerea, Cercidium floridium, Olneya tesota, Lycium californicum, Merremia aurea, Esenbeckia flava, Haematoxylon brasiletto, Yucca valida, Pithecellobium confine, Krameria parvifolia, Ferocactus peninsulae, Prosopis juliflora, Pachycereus pringlei y algunos pastos de los géneros Bouteloua babata, Aristida sp, Hyptis sp. (Morelos 1988). La capacidad del área de pastoreo es de 40 hectáreas por unidad animal (COTECOCA 1975), considerando como unidad animal a una vaca adulta de $454 \mathrm{~kg}$ con su cría hasta que ésta sea destetada (Gutiérrez 1991).

\section{DISEÑO DE TRATAMIENTOS Y MANEJO DE LOS ANIMALES}

En el hato A se utilizaron 60 vacas Charolais adultas y en el hato B 60 vacas Chinampas (Criollo local) adultas. En cada una de las dos explotaciones las vacas se distribuyeron aleatoriamente en tres tratamientos $(n=20$ vacas por tratamiento). Los tratamientos aplicados en ambos hatos radicaron en diferentes suplementos alimenticios proporcionados a las vacas dentro de la temporada de sequía (2 kg/vaca/ día). El tratamiento testigo (TE) en cada hato consumió un suplemento sin grasa adicional; otro grupo consumió un suplemento que contenía 9,6\% de sebo de bovino (SB) el cual es permitido en México para la alimentación de bovinos; y el tercero (JCAG), un suplemento con 9,8\% de una grasa protegida de la degradación ruminal en forma de jabones de calcio de ácidos grasos (Megalac ${ }^{\circledR}$, Church \& Dwight Co., Inc., Princeton, NJ). La composición de los suplementos alimenticios se muestra en el cuadro 1 . Dichos suplementos fueron proporcionados diariamente de manera individual cuando las vacas se acercaban al sitio de abrevadero. Los tres grupos de animales dentro de cada hato compartieron la misma área de pastoreo (2.000 hectáreas, aproximadamente en cada localidad). Los suplementos se proporcionaron durante 90 días, iniciando 30 días antes del parto, aproximadamente. Las vacas fueron sometidas a un programa de apareamiento natural continuo, utilizando una relación de un semental por cada 20 hembras. Todos los sementales (Brahman en ambos hatos) fueron sometidos a un examen físico y a una prueba de fertilidad del semen antes de iniciar el periodo de montas.

\section{MEDICIONES}

Los valores de las variables climáticas registradas durante los 90 días del estudio fueron tomados de la estación meteorológica más cercana al sitio de trabajo, la cual se encontraba a $2 \mathrm{~km}$, aproximadamente.

Se registró el peso de las crías al nacimiento y a los 35 y 90 días de edad en promedio y se cuantificó la producción de leche de las vacas a los 35 y 90 días del periodo de lactación. Para esto se utilizó la técnica de pesaje y amamantamiento del ternero descrita por Espinoza y Ríos (1985). Esta técnica consiste en la separación de las vacas de sus crías a las 18:00 horas, quedando los becerros en un corral y sus madres pasaban a un potrero. Al día siguiente (06:00 horas) las vacas y sus crías se reunían permitiendo el amamantamiento durante 30 minutos considerando que con esto la ubre quedaba completamente vacía. Se separaban nuevamente los terneros de sus madres y a las 18:00 horas de ese día se pesaban antes y después de un amamantamiento de 30 minutos. La diferencia en el peso de los terneros antes y después del amamantamiento se consideró la producción de leche en un periodo de 12 horas. Una vez más se hacía la separación de los animales y a las 06:00 horas del día siguiente se repetía el procedimiento. La suma de las diferencias de peso de los terneros en cada ocasión se consideró como la producción de leche en un lapso de 24 horas. Se registraron el peso y la condición corporal de las vacas al parto y a los 90 días posparto utilizando para la condición corporal la escala establecida por Whitman (1975). El diagnóstico de preñez 
Cuadro 1. Composición y nutrientes de los suplementos, por tratamiento.

Composition and nutrients of supplements, by treatment.

\begin{tabular}{lccc}
\hline \multirow{2}{*}{ Componente (\%) } & & Suplemento $^{1}$ & SB $^{4}$ \\
\cline { 2 - 4 } Sebo de bovino & $\mathrm{TE}^{2}$ & $\mathrm{JCAG}^{3}$ & 9,5 \\
Megalac & 0 & 0 & 0 \\
Salvado de trigo & 0 & 9,6 & 44,1 \\
Maíz molido & 9,9 & 37,2 & 16,6 \\
Trigo molido & 23,4 & 19,6 & 15,0 \\
Harina de soya & 44,5 & 15,0 & 2,8 \\
Semilla de algodón & 7,5 & 2,8 & 1,9 \\
Urea & 2,8 & 5,7 & 0,87 \\
Melaza & 0,4 & 0,84 & 8,9 \\
\hline Composición química & 11,1 & 8,9 & 3,2 \\
\hline EM (Mcal/kg) & & & 16,6 \\
Proteína cruda (\%) & 3,0 & 3,1 & 13,4 \\
Grasa (\%) & 16,6 & 16,6 & 13,2 \\
\hline
\end{tabular}

1 Los valores mostrados fueron calculados en base a materia seca.

2 Suplemento sin grasa adicional.

3 Suplemento con jabones de calcio de ácidos grasos (Megalac ${ }^{\circledR}$, Church \& Dwight Co., Inc., Princeton, NJ; contiene $80 \%$ de grasa y $8 \%$ de calcio).

4 Sebo de bovino.

5 Energía metabolizable, calculada de NRC (1984).

lo realizó un especialista en reproducción bovina mediante palpación rectal.

\section{MUESTREOS SANGUÍNEOS Y TÉCNICAS DE LABORATORIO}

El día 60 posparto en el hato $\mathrm{B}$, se colectaron muestras de sangre $(10 \mathrm{ml})$ de cada vaca mediante punción de la vena yugular y utilizando tubos vacutainer sin anticoagulante. Las muestras se refrigeraron a $4{ }^{\circ} \mathrm{C}$, se centrifugaron (1500 x $g$ durante 15 minutos) dentro de los 60 minutos posteriores a la colección y el suero se almacenó en tubos de plástico estériles a $-20{ }^{\circ} \mathrm{C}$ hasta que los metabolitos de lípidos (colesterol total, lipoproteínas de alta densidad, lipoproteínas de baja densidad y triglicéridos) fueron cuantificados por medio de procedimientos enzimáticos automatizados (Espinoza y col 1995). El colesterol total se determinó en el suero después de haber sido hidrolizado y oxidado enzimáticamente. En el proceso de oxidación se produjo $\mathrm{H}_{2} \mathrm{O}_{2}$. En presencia de peroxidasa, reaccionando con 4-amino-antipirina y fenol, se transformó en un colorante de quinonimina (Merck-México, S. A.). Las lipoproteínas de alta densidad se separaron de los quilomicrones y lipoproteínas de baja densidad mediante la adición de un reactivo precipitante al suero. Después de la centrifugación, el contenido de colesterol total y la fracción de lipoproteínas de alta densidad permanecen en el sobrenadante y es determinada por el método colorimétrico enzimático utilizando colesterol estearasa, colesterol oxidasa, peroxidasa y el cromógeno 4-aminofenasona/ fenol (Sera-Pak, Ames Division). Las lipoproteínas de baja densidad se determinaron por diferencia del colesterol total y lipoproteínas de alta densidad. El método colorimétrico enzimático para la determinación de TG está basado en el principio de que el glicerol liberado de la hidrólisis de triglicéridos por la lipoproteína lipasa es convertido por la gliceroquinasa a glicerol-3-fosfato, el cual es oxidado por la glicerofosfato oxidasa a dihidroxiacetona fosfato y $\mathrm{H}_{2} \mathrm{O}_{2}$. En presencia de peroxidasa, el $\mathrm{H}_{2} \mathrm{O}_{2}$ oxida el cromógeno 4-aminofenasona/N-etil-N-(3-sulfopropil)$\mathrm{m}$-anisidina a un compuesto de color violeta (Sera-Pak, Ames Division).

\section{ANÁLISIS ESTADÍSTICO}

El peso de las crías al nacimiento y a los 35 y 90 días, condición corporal, peso de las vacas y la producción de leche se examinaron dentro de cada hato mediante un análisis de varianza utilizando el procedimiento GLM de SAS (SAS 1989). El mismo procedimiento se utilizó para el análisis de los metabolitos de lípidos (hato B). El modelo estadístico utilizado fue:

$$
\mathrm{Y}_{i j}=\mu+\tau_{i}+\varepsilon_{i j}
$$

Donde:

$\mathrm{Y}_{i j}$ corresponde a cada una de las variables dependientes;

$\tau_{i}$ es el efecto del i-ésimo tratamiento; $\varepsilon_{i j}$ es el error experimental. 
Las medias se compararon utilizando contrastes ortogonales (TE vs SB+JCAG y SB vs JCAG). Los porcentajes de preñez se compararon entre tratamientos dentro de cada hato mediante una prueba de Chi cuadrado para homogeneidad de parámetros (SAS 1989).

\section{RESULTADOS}

\section{CONDICIONES CLIMÁTICAS}

Las condiciones climáticas promedio que prevalecieron durante el periodo en que se desarrolló el estudio fueron relativamente estables. La velocidad del viento varió de 8,25 a 9,4 metros/segundo. El grado de insolación, medido como la cantidad de horas con sol durante el día, fluctuó entre 7,13 y 11,47 horas. La temperatura máxima durante el día fluctuó entre 36,2 y $40,3{ }^{\circ} \mathrm{C}$ y la humedad relativa varió de 69,8 a $85 \%$. La precipitación pluvial acumulada en los 90 días de suplementación fue de $75 \mathrm{~mm}$.

\section{PRODUCCIÓN DE LECHE}

Los valores de producción de leche observados en ambos hatos se expresan en el cuadro 2. La producción de leche a los 35 días de lactación en el hato A fue mayor en las vacas que recibieron suplementación de grasas $(\mathrm{P}<0,05)$ que en las del grupo testigo (TE), pero no hubo diferencia significativa en la producción de leche de las vacas suplementadas con distintos tipos de lípidos $(\mathrm{P}>0,05)$. A los 90 días de lactación, la producción de leche también fue mayor en las vacas suplementadas con grasa $(\mathrm{P}<0,01)$, sin registrarse diferencia significativa $(\mathrm{P}>0,05)$ entre las que consumieron el sebo de bovino (SB) o la grasa protegida de la degradación en el rumen (JCAG).

En el hato B, la producción de leche a los 35 días posparto fue similar entre las vacas del grupo TE y las que se suplementaron con algún tipo de grasa $(P>0,05)$. En este mismo periodo, las vacas del tratamiento JCAG produjeron más leche que las del tratamiento SB $(\mathrm{P}<0,05)$. A los 90 días de la lactación la producción de leche resultó similar entre los tratamientos $(\mathrm{P}>0,05)$.

\section{PESO DE LAS CRÍAS}

En el cuadro 3 se puede observar que en ambos hatos el peso de las crías a diferentes edades siguió una tendencia parecida. Los pesos al nacimiento fueron similares entre los terneros del tratamiento $\mathrm{TE}$ con respecto a $\mathrm{SB}+\mathrm{JCAG}$ y entre SB vs JCAG $(\mathrm{P}>0,05)$. A los 35 días de edad, los terneros en TE fueron más livianos $(\mathrm{P}<0,01)$ que aquellos pertenecientes a los tratamientos que recibieron suplemento de grasa ( $\mathrm{SB}+\mathrm{JCAG})$. No se registró diferencia significativa en el peso de las crías a los 35 días de edad por efecto del tipo de grasa suplementada a sus madres $(\mathrm{P}>0,05)$. El peso de los terneros a los 90 días de edad resultó menor en TE que en $\mathrm{SB}+\mathrm{JCAG}(\mathrm{P}<0,01)$ pero similar entre SB y JCAG $(\mathrm{P}>0,05)$.

\section{PESO Y CONDICIÓN CORPORAL DE LAS VACAS}

Los valores de condición y peso corporal de las vacas en ambos hatos se muestran en el cuadro 4. Tanto en el hato A como en el hato B la condición y el peso corporal al parto fueron similares entre los tratamientos TE y $\mathrm{SB}+\mathrm{JCAG}$ y entre las vacas suplementadas con sebo de bovino o grasa protegidas de la digestión ruminal $(\mathrm{P}>0,05)$. En ambos hatos la condición corporal a los 90 días posparto fue mayor en las vacas que consumieron grasa $(\mathrm{SB}+\mathrm{JCAG})$ que en las del tratamiento $\mathrm{TE}$ $(\mathrm{P}<0,01)$ y no hubo diferencia entre las vacas de los tratamientos SB y JCAG $(\mathrm{P}>0,05)$. El peso corporal a los 90 días posparto fue menor en las vacas de TE con respecto a $\mathrm{SB}+\mathrm{JCAG}$ en el hato $\mathrm{A}(\mathrm{P}<0,05)$ y en el hato $\mathrm{B}(\mathrm{P}<0,01)$ y en ninguno de los hatos hubo diferencia entre los tratamientos SB y JCAG (P > 0,05). La pérdida de condición corporal (PeCC) durante los primeros 90 días de lactación siguió la misma tendencia en los dos hatos $\mathrm{y}$ fue mayor en las vacas del tratamiento TE que en las que se suplementaron con grasa $(\mathrm{P}<0,01)$ pero similar entre los tratamientos SB y JCAG $(\mathrm{P}>0,05)$. Las vacas del tratamiento TE perdieron más peso $(\mathrm{P}<0,01)$ que las que se suplementaron con grasa $(\mathrm{SB}+\mathrm{JCAG})$ y no hubo diferencia significativa en la PeCC $(\mathrm{P}>0,05)$ atribuible al tipo de grasa suplementada (SB vs JCAG).

\section{EFICIENCIA REPRODUCTIVA}

En el hato A, los porcentajes de preñez fueron de 45,70 y $80 \%$ y en el hato B de 25,55 y $58 \%$ en las vacas de los tratamientos TE, JCAG y SB, respectivamente. En ambos hatos, el porcentaje de preñeces fue superior $(\mathrm{P}<0,05)$ en los animales que consumieron los suplementos conteniendo grasa protegida de la digestión ruminal o el sebo de bovino, resultando estos similares entre sí $(\mathrm{P}>0,05)$.

\section{METABOLITOS DE LOS LÍPIDOS}

En el cuadro 5 se observa que las concentraciones séricas de colesterol total, lipoproteínas de alta densidad, lipoproteínas de baja densidad y de triglicéridos en las vacas del hato $B$ fueron más elevadas en las vacas que se suplementaron con grasa (SB + JCAG) que los animales del tratamiento TE $(\mathrm{P}<0,01)$. Ninguno de los metabolitos se vio afectado en su concentración sérica por el tipo de grasa suplementada $(\mathrm{P}>0,05)$.

\section{DISCUSIÓN}

\section{PRODUCCIÓN DE LECHE}

Como ha podido observarse, en el presente trabajo hubo un efecto de la suplementación de grasas sobre la producción de leche en uno de los hatos (A). No existen reportes relacionados con la suplementación de grasas a 
Cuadro 2. Producción de leche (media \pm EE) a los 35 (PL35) y a los 90 (PL90) días de lactación, por tratamiento, dentro de cada hato.

Milk yield (mean \pm SE) at 35 (PL35) and 90 (PL90) days of lactation, by treatment, within herd.

\begin{tabular}{|c|c|c|c|c|c|c|c|c|c|c|}
\hline \multirow[b]{2}{*}{ Variable } & \multicolumn{3}{|c|}{ Tratamientos en el hato $\mathrm{A}^{\mathrm{a}}$} & \multicolumn{2}{|c|}{$\begin{array}{c}\text { Contraste, } \\
\text { valor de la } \mathrm{P}^{\mathrm{b}}\end{array}$} & \multicolumn{3}{|c|}{ Tratamientos en el hato $\mathrm{B}^{\mathrm{a}}$} & \multicolumn{2}{|c|}{$\begin{array}{c}\text { Contraste, } \\
\text { valor de la } \mathrm{P}^{\mathrm{b}}\end{array}$} \\
\hline & TE & SB & JCAG & (1) & (2) & TE & SB & JCAG & $(1)$ & $(2)$ \\
\hline PL35 (kg) & $4,0 \pm 0,13$ & $4,6 \pm 0,12$ & $4,7 \pm 0,10$ & 0,04 & 0,06 & $3,2 \pm 0,26$ & $3,1 \pm 0,26$ & $3,8 \pm 0,26$ & 0,07 & 0,03 \\
\hline PL90 (kg) & $3,3 \pm 0,08$ & $4,0 \pm 0,07$ & $3,9 \pm 0,09$ & 0,00 & 0,34 & $3,9 \pm 0,16$ & $3,6 \pm 0,19$ & $3,9 \pm 0,20$ & 0,43 & 0,26 \\
\hline
\end{tabular}

a $\mathrm{TE}=$ suplemento testigo, sin grasa adicional; $\mathrm{SB}=$ suplemento conteniendo sebo de bovino; JCAG = suplemento conteniendo jabones de calcio de ácidos grasos (Megalac ${ }^{\circledR}$, Church \& Dwight Co., Inc., Princeton, NJ; contiene 80\% de grasa y 8\% de calcio).

b Los contrastes fueron: (1) TE vs SB + JCAG; (2) SB vs JCAG.

Cuadro 3. Peso de las crías (media \pm EE) al nacimiento (PC0), a los 35 (PC35) y a los 90 (PC90) días de edad, por tratamiento, dentro de cada hato.

Weight of calves (mean \pm SE) at birth (PC0), at 35 (PV35) and 90 (PC90) days of age, by treatment, within herd.

\begin{tabular}{|c|c|c|c|c|c|c|c|c|c|c|}
\hline \multirow[b]{2}{*}{ Variable } & \multicolumn{3}{|c|}{ Tratamientos en el hato $\mathrm{A}^{\mathrm{a}}$} & \multicolumn{2}{|c|}{$\begin{array}{c}\text { Contraste, } \\
\text { valor de la } \mathrm{P}^{\mathrm{b}}\end{array}$} & \multicolumn{3}{|c|}{ Tratamientos en el hato $\mathrm{B}^{\mathrm{a}}$} & \multicolumn{2}{|c|}{$\begin{array}{c}\text { Contraste, } \\
\text { valor de la } \mathrm{P}^{\mathrm{b}}\end{array}$} \\
\hline & $\mathrm{TE}$ & SB & JCAG & (1) & (2) & $\mathrm{TE}$ & SB & JCAG & (1) & (2) \\
\hline $\mathrm{PC0}(\mathrm{kg})$ & $31,5 \pm 0,4$ & $32,5 \pm 0,5$ & $32,0 \pm 0,4$ & 0,07 & 0,93 & $23,5 \pm 0,5$ & $23,0 \pm 0,3$ & $23,2 \pm 0,3$ & 0,37 & 0,75 \\
\hline PC35 (kg) & $53,3 \pm 0,7$ & $55,6 \pm 0,7$ & $55,6 \pm 0,7$ & 0,00 & 0,19 & $33,1 \pm 0,7$ & $38,1 \pm 0,8$ & $36,0 \pm 0,7$ & 0,00 & 0,05 \\
\hline PC90 (kg) & $62,3 \pm 0,7$ & $70,1 \pm 0,9$ & $69,8 \pm 0,8$ & 0,00 & 0,81 & $51,4 \pm 1,5$ & $60,3 \pm 1,0$ & $57,0 \pm 1,1$ & 0,00 & 0,06 \\
\hline
\end{tabular}

a $\mathrm{TE}=$ suplemento testigo, sin grasa adicional; $\mathrm{SB}=$ suplemento conteniendo sebo de bovino; JCAG = suplemento conteniendo jabones de calcio de ácidos grasos (Megalac ${ }^{\circledR}$, Church \& Dwight Co., Inc., Princeton, NJ; contiene 80\% de grasa y 8\% de calcio).

b Los contrastes fueron: (1) TE vs SB + JCAG; (2) SB vs JCAG.

Cuadro 4. Condición corporal y peso de las vacas (media \pm EE) al parto y a los 90 días posparto, por tratamiento, dentro de cada hato.

Body condition score and body weight of cows (mean \pm SE) at calving and 90 days postcalving, by treatment, within herd.

\begin{tabular}{|c|c|c|c|c|c|c|c|c|c|c|}
\hline \multirow[b]{2}{*}{ Variable $^{c}$} & \multicolumn{3}{|c|}{ Tratamientos en el hato $\mathrm{A}^{\mathrm{a}}$} & \multicolumn{2}{|c|}{$\begin{array}{c}\text { Contraste, } \\
\text { valor de la } \mathrm{P}^{\mathrm{b}}\end{array}$} & \multicolumn{3}{|c|}{ Tratamientos en el hato $\mathrm{B}^{\mathrm{a}}$} & \multicolumn{2}{|c|}{$\begin{array}{l}\text { Contraste, } \\
\text { valor la } \mathrm{P}^{\mathrm{b}}\end{array}$} \\
\hline & $\mathrm{TE}$ & SB & JCAG & (1) & (2) & $\mathrm{TE}$ & SB & JCAG & (1) & (2) \\
\hline $\mathrm{CCP}$ & $5,5 \pm 0,13$ & $5,2 \pm 0,15$ & $5,4 \pm 0,13$ & 0,15 & 0,22 & $5,7 \pm 0,11$ & $5,4 \pm 0,13$ & $5,5 \pm 0,14$ & 0,10 & 0,58 \\
\hline CC90 & $4,08 \pm 0,12$ & $4,45 \pm 0,09$ & $4,55 \pm 0,07$ & 0,00 & 0,47 & $3,0 \pm 0,14$ & $3,9 \pm 0,13$ & $4,0 \pm 0,11$ & 0,00 & 0,56 \\
\hline $\mathrm{PCP}(\mathrm{kg})$ & $386,1 \pm 6,2$ & $395,6 \pm 6,4$ & $387,5 \pm 6,3$ & 0,51 & 0,35 & $315 \pm 5,2$ & $319 \pm 5,3$ & $317 \pm 5,4$ & 0,63 & 0,82 \\
\hline PC90 (kg) & $340,8 \pm 4,8$ & $358,6 \pm 4,9$ & $353,5 \pm 4,6$ & 0,01 & 0,41 & $260 \pm 6,2$ & $290 \pm 6,3$ & $295 \pm 6,4$ & 0,00 & 0,60 \\
\hline $\mathrm{PeCC}$ & $-1,42 \pm 0,07$ & $-0,75 \pm 0,07$ & $-0,85 \pm 0,06$ & 0,00 & 0,32 & $-2,7 \pm 0,08$ & $-1,5 \pm 0,09$ & $-1,5 \pm 0,08$ & 0,00 & 0,99 \\
\hline
\end{tabular}

a $\mathrm{TE}=$ suplemento testigo, sin grasa adicional; $\mathrm{SB}=$ suplemento conteniendo sebo de bovino; JCAG = suplemento conteniendo jabones de calcio de ácidos grasos $\left(\right.$ Megalac $^{\circledR}$, Church \& Dwight Co., Inc., Princeton, NJ; contiene $80 \%$ de grasa y $8 \%$ de calcio).

b Los contrastes fueron: (1) TE vs SB + JCAG; (2) SB vs JCAG.

c $\mathrm{CCP}=$ condición corporal al parto; $\mathrm{CC} 90=$ condición corporal a los 90 días posparto; $\mathrm{PCP}=$ peso corporal al parto; $\mathrm{PC} 90=$ peso corporal a los 90 días posparto; $\mathrm{PeCC}=$ pérdida de condición corporal entre el parto y los 90 días posparto.

vacas para carne en donde se haya cuantificado la producción de leche. No obstante, las grasas protegidas de la digestión ruminal (Sklan y col 1991, Moallem y col 2000, 2007) y el sebo de bovino (Bobe y col 2009) han incrementado la producción de leche en vacas Holstein; las grasas protegidas, presumiblemente debido a efectos extrarruminales y el sebo de bovino debido posiblemente a su efecto en la fermentación ruminal y la consecuente influencia en la producción de ácidos grasos volátiles (Sklan y col 1992). Es probable que en la presente investigación 
Cuadro 5. Medias ( $\pm \mathrm{EE}$ ) para la concentración sérica de metabolitos de lípidos en las vacas del hato B, por tratamiento. Means $( \pm$ SE) of serum concentration of lipid metabolites in cows of herd B, by treatment.

\begin{tabular}{|c|c|c|c|c|c|}
\hline \multirow[b]{2}{*}{$\begin{array}{l}\text { Metabolito } \\
(\mathrm{mg} / \mathrm{dl})^{\mathrm{c}}\end{array}$} & \multicolumn{3}{|c|}{ Tratamientos } & \multicolumn{2}{|c|}{ Contraste, valor de la $\mathrm{P}^{\mathrm{b}}$} \\
\hline & $\mathrm{TE}$ & SB & JCAG & (1) & (2) \\
\hline $\mathrm{COL}$ & $130 \pm 6,9$ & $160,2 \pm 7,2$ & $165,8 \pm 6,4$ & 0,00 & 0,52 \\
\hline LAD & $60,5 \pm 4,1$ & $90,1 \pm 4,5$ & $95,7 \pm 4,6$ & 0,00 & 0,28 \\
\hline LBD & $74,8 \pm 5,2$ & $100,4 \pm 5,1$ & $102,6 \pm 4,9$ & 0,00 & 0,74 \\
\hline TG & $98 \pm 5,4$ & $140 \pm 5,7$ & $138,7 \pm 5,1$ & 0,00 & 0,86 \\
\hline
\end{tabular}

las vacas que consumieron grasa adicional en el suplemento hayan tenido un mejor balance energético ya que el contenido de energía metabolizable fue de 3,0, 3,1 y 3,2 Mcal/kg en TE, JCAG y SB, respectivamente. Lo anterior concuerda con el resultado obtenido por Erickson y col (1992) en vacas Holstein suplementadas con jabones calcio de ácidos grasos en una dieta cuyo contenido energético era ligeramente superior al del grupo testigo. Los resultados observados en el hato B están de acuerdo con los constatados en vacas Holstein (Boken y col 2005) y Lucerna de Colombia (Mahecha y col 2008) que fueron alimentadas con dietas que contenían grasas protegidas de la digestión ruminal y con las observaciones de López y col (2007) en vacas Jersey suplementadas con sebo de bovino o grasa de escape ruminal.

\section{PESO DE LAS CRÍAS}

El peso superior de los terneros a los 35 y 90 días de edad en los tratamientos que se suplementaron con grasa $(\mathrm{SB}+\mathrm{JCAG})$ en el hato A pudiera estar relacionado con la mayor producción de leche de las vacas en dichos periodos (Coppock y Wilks 1991) ya que las crías consumieron de 500 a 600 g más de leche por día en comparación con el tratamiento TE. Aunque en el hato B la producción de leche fue estadísticamente similar entre los tratamientos, al menos a los 35 días de edad los terneros de las vacas suplementadas con grasa adicional (SB + JCAG) consumieron alrededor de $600 \mathrm{~g}$ más de leche por día, comparados con los del grupo testigo, esto podría explicar también la diferencia en el peso de los terneros en el periodo mencionado. Pesos superiores a los 35, 50 y 95 días de edad en los terneros de vacas suplementadas con grasa protegida de la digestión en el rumen (con respecto a los del grupo testigo) han sido observados también en bovinos Angus y Hereford x Angus (Espinoza y col 1995).

\section{PESO Y CONDICIÓN CORPORAL DE LAS VACAS}

La CC observada al parto en las vacas del presente trabajo concuerda con un estudio realizado en vacas Angus y Hereford x Angus adultas en el que los animales suplementados con jabones de calcio de ácidos grasos desde los 60 días preparto registraron CC similar al tratamiento testigo (Espinoza y col 1995). En ese estudio, la CC a los 35 y 50 días posparto resultó mayor en las vacas que consumieron grasa adicional pero, a diferencia de lo observado en la presente investigación, la CC a los 90 días de lactación fue similar entre los animales suplementados con grasa y los del tratamiento testigo. Esta discrepancia en la CC a los 90 días con respecto al presente estudio se pudiera deber a que en la presente investigación las vacas pasaron sus primeros tres meses posparto durante la temporada de sequía, en cambio en el estudio de Espinoza y col (1995) la disponibilidad de forraje en el agostadero se incrementó a partir de los 60 días posparto, aproximadamente. La diferencia de la CC entre el grupo testigo y los que consumieron grasa adicional también pudiera explicarse con base en otros estudios que han observado que la concentración sérica de insulina aumentó en ovejas que consumieron sebo de bovino o jabones de calcio de ácidos grasos (Espinoza y col 2008) y un mecanismo para el incremento de la adipogénesis puede ser a través de la activación de receptores importantes en la diferenciación de preadipocitos a adipocitos por parte de dicha hormona (Kokta y col 2008).

\section{EFICIENCIA REPRODUCTIVA Y METABOLITOS DE LOS} LÍPIDOS

La investigación sobre la suplementación de grasas ha dado resultados inconsistentes y variados en relación a la eficiencia reproductiva, incluyendo efectos positivos, 
negativos y efectos no aparentes (Funston 2004). Sin embargo, los resultados de este estudio concuerdan con otros en los que el porcentaje de vacas preñadas en la primera mitad de la temporada de apareamientos fue superior en los animales que consumieron grasa protegida de la digestión ruminal a partir de los 60 días preparto (Espinoza y col 1995); concuerdan también con un reporte de Hess y col (2005) cuyas evidencias revelan un mejoramiento en las tasas de preñez en vacas de carne suplementadas con grasa durante la última etapa de la gestación.

La utilización de dos dietas hiperlipídicas distintas (21 y $17 \%$ de grasa; $1,8 \mathrm{~kg}$ /día) mejoró la presentación de estros en vacas de dos años de edad y las tasas de concepción al primer servicio en vacas maduras cuando se suplementaron durante 51 y 45 días posparto, respectivamente, en comparación del grupo testigo que consumió un $1,8 \mathrm{~kg} / \mathrm{día}$ de un suplemento con 3\% de grasa (Bader y col 2000).

Una situación apropiada para la suplementación de grasa puede ocurrir cuando las pasturas en el sitio de pastoreo son limitadas ya que la utilización de lípidos adicionales en el suplemento de vaquillas bien desarrolladas o en vacas con una condición corporal adecuada pastoreando en áreas con suficientes recursos forrajeros podría no tener beneficios más allá del aporte de energía en la dieta (Funston 2004).

La suplementación de grasas en el ganado bovino causa una serie de cambios metabólicos que pudieran relacionarse con las funciones reproductivas que dan lugar a mayores tasas de preñez, como es el incremento en las concentraciones basales de insulina en vacas para carne (Thomas y Williams 1996). Este fenómeno se ha observado en vacas ciclando y en respuesta a la suplementación con grasa durante el periodo posparto (Williams y Stanko 2000). Las concentraciones séricas de insulina también se incrementan gradualmente en respuesta a otro tipo de grasas como el sebo de bovino (Thomas y Williams 1996). Las mayores tasas de preñez observadas en el presente estudio podrían relacionarse con una mayor viabilidad embrionaria en las vacas suplementadas con grasas ya que se ha demostrado que el uso de jabones de calcio de ácidos grasos en vacas lecheras provoca el desarrollo de blastocistos con una mayor cantidad de células, tanto en su masa interna como en el trofoectodermo (Fouladi-Nashta y col 2007).

Coincidiendo con los resultados del presente trabajo, estudios previos indican que las grasas protegidas de la degradación ruminal en bovinos para carne (Espinoza y col 1995) y en ovejas (Ghoreishi y col 2007) y el consumo de sebo de bovino en ovejas (Espinoza y col 1998) promueven un aumento en los niveles plasmáticos de colesterol, lipoproteínas de alta densidad, lipoproteínas de baja densidad y triglicéridos. La suplementación de grasa en rumiantes incrementa marcadamente el transporte de lipoproteínas a partir del intestino delgado aportando el sustrato para la síntesis de progesterona durante la fase lútea del ciclo estral (Hawkins y col 1995), contribuyendo con ello a una mayor calidad y capacidad de desarrollo del embrión (Santos y col 2008). Algunos ácidos grasos poliinsaturados (eicosapentaenoico y docosahexaenoico) pueden favorecer la fertilidad del ganado suprimiendo la síntesis de PGF2-alfa al regular la expresión de algunos genes implicados en la biosíntesis de la hormona señalada (Coyne y col 2008).

Las evidencias anteriores podrían explicar al menos de manera parcial los beneficios del consumo de grasa en bovinos sobre las tasas de preñez si se considera que tanto las lipoproteínas de alta densidad como las lipoproteínas de baja densidad promueven in vitro la viabilidad de las células de la granulosa de folículos en distintas etapas de desarrollo, incrementando la proliferación de las células de la teca provenientes de folículos preovulatorios y estimulan la producción de IGF-I por la granulosa y células de la teca (Williams y Stanko 2000).

De acuerdo con los resultados obtenidos en el presente estudio se concluye que la suplementación durante el periodo de sequía de grasa bovina y de grasa protegida de la digestión ruminal en la dieta de vacas para carne en condiciones de pastoreo incrementó la producción de leche durante el primer tercio de la lactación, el crecimiento de los terneros, la condición corporal de las vacas reduciendo su pérdida en el periodo posparto, los niveles séricos de los metabolitos de los lípidos y las tasas de preñez.

\section{RESUMEN}

Con el fin de evaluar el efecto de la suplementación con dos fuentes de grasa en vacas para carne en pastoreo se utilizaron 120 hembras adultas en dos hatos. De manera independiente, en un hato (A) con 60 vacas Charolais y en otro (B) con 60 vacas Chinampas (criollo local) se suplementaron (2 $\mathrm{kg} / \mathrm{vaca} /$ día) concentrados isoproteicos $(\mathrm{PC}=16,6 \%)$ con un contenido similar de $\mathrm{EM}(\approx 3,0 \mathrm{Mcal} / \mathrm{kg})$ durante 90 días (iniciando 30 días preparto). En cada hato, las vacas se dividieron aleatoriamente en tres grupos. Los grupos testigo (TE; $\mathrm{n}=20$ ) recibieron suplemento con $3,2 \%$ de grasa. Otro grupo de 20 vacas en cada hato recibió un suplemento conteniendo grasa adicional $(13,2 \%)$ en forma de jabones de calcio de ácidos grasos (JCAG). El tercer grupo $(\mathrm{SB})$ dentro de cada hato $(\mathrm{n}=20)$ consumió suplemento con $13,4 \%$ de grasa a base de sebo de bovino. Para la comparación de las medias en las variables no categóricas se utilizaron contrastes ortogonales (TE vs SB + JCAG y SB vs JCAG). En el hato A, la producción de leche fue mayor a los $35(\mathrm{P}<0,05)$ y a los $90(\mathrm{P}<0,01)$ días de la lactación en las vacas que se suplementaron con grasa $(\mathrm{SB}+\mathrm{JCAG})$ que en las del tratamiento TE. En ambos hatos, el peso de los terneros a los 35 y 90 días de edad y el peso y condición corporal de las vacas a los 90 días posparto fueron mayores en $\mathrm{SB}+\mathrm{JCAG}$ que en $\mathrm{TE}(\mathrm{P}<0,01)$. Las concentraciones séricas de colesterol, lipoproteínas de alta densidad, lipoproteínas de baja densidad y triglicéridos fueron superiores en las vacas suplementadas con grasas $(\mathrm{SB}+\mathrm{JCAG})$ que en las del tratamiento TE $(\mathrm{P}<0,01)$. Las tasas de preñez en ambos hatos fueron mayores en las vacas que consumieron grasa adicional en el suplemento $(\mathrm{P}<0,05)$. Se concluye que la grasa adicional incorporada en un suplemento durante los periodos pre y posparto de vacas para carne en pastoreo incrementó la producción de leche, el crecimiento de los terneros, el peso y condición corporal de las vacas, los metabolitos de los lípidos y las tasas de preñez.

\section{REFERENCIAS}

Auchtung TL, DJ Baer, RA Erdman, SM Barao, GE Dahl. 2002. Relation of growth hormone response to growth hormone-releasing hormone 
to estimation of milk production via deuterium oxide dilution in beef cattle. J Anim Sci 80, 1270-1274.

Bader JF, EED Felton, MS Kerley, DD Simms, DJ Patterson. 2000. Effects of postpartum fat supplementation on reproduction in primiparous 2-year-old and mature cows. J Anim Sci 78 (Suppl 1), 224.

Bobe G, GL Lindberg, LF Reutzel, MD Hanigan. 2009. Effects of lipid supplementation on the yield and composition of milk from cows with different beta-lactoglobulin phenotypes. J Dairy Sci 92, 197-203.

Boken SL, CR Staples, LE Sollenberger, TC Jenkins, WW Thatcher. 2005. Effect of grazing and fat supplementation on production and reproduction of Holstein cows. J Dairy Sci 88, 4258-4272.

Bottger JD, BW Hess, BM Alexander, DL Hixon, LF Woodard, RN Funston, DM Hallford, GE Moss. 2002. Effects of supplementation with high linoleic or oleic cracked safflower seeds on postpartum reproduction and calf performance of primiparous beef heifers. J Anim Sci 80, 2023-2030.

Coppock CE, DL Wilks. 1991. Supplemental fat in high energy rations for lactating cows: Effects on intake, digestion, milk yield, and composition. J Anim Sci 69, 3826-3837.

COTECOCA, Coeficientes de Agostadero de la República Mexicana 1975. Estado de Baja California Sur. Secretaría de Agricultura y Ganadería, México D. F., México.

Coyne GS, DA Kenny, S Childs, JM Sreenan, SM Waters. 2008. Dietary n-3 polyunsaturated fatty acids alter the expression of genes involved in prostaglandin biosynthesis in the bovine uterus. Theriogenology 70, 772-782.

DGTENAL, Dirección General de Geografía del Territorio Nacional. 1980. Carta Topográfica. La Paz, Baja California Sur, México.

Erickson PS, MR Murphy, JH Clark. 1992. Supplementation of dairy cows diets with calcium salts of long-chain fatty acids and nicotinic acid in early lactation. J Dairy Sci 75, 1078-1089.

Espinoza JL, JG Ríos. 1985. Producción de leche de vacas Hereford, Angus y su cruza, bajo condiciones de agostadero. Rev Mex Prod Animal 17, 3-11.

Espinoza JL, JA Ramírez-Godínez, JA Jiménez, A Flores. 1995. Effects of calcium soaps of fatty acids on postpartum reproductive activity in beef cows and growth of calves. J Anim Sci 73, 2888-2892.

Espinoza JL, O López-Molina, JA Ramírez-Godínez, J Jiménez, A Flores. 1998. Milk composition, postpartum reproductive activity and growth of lambs in Pelibuey ewes fed calcium soaps of long chain fatty acids. Small Ruminant Res 27, 119-124.

Espinoza JL, A Palacios, R Ortega, A Guillén. 2008. Efecto de la suplementación de grasas sobre las concentraciones séricas de progesterona, insulina, somatotropina y algunos metabolitos de los lípidos en ovejas Pelibuey. Arch Med Vet 40, 135-140.

Fouladi-Nashta AA, CG Gutierrez, JG Gong, PC Garnsworthy, R Webb. 2007. Impact of dietary fatty acids on oocyte quality and development in lactating dairy cows. Biol Reprod 77, 9-17.

Freetly HC, JA Nienaber, T Brown-Brandl. 2006. Partitioning of energy during lactation of primiparous beef cows. J Anim Sci 84, 2157-2162.

Funston RN. 2004. Fat supplementation and reproduction in beef females. J Anim Sci 82 (Suppl E), E154-E161.

Ghoreishi SM, MJ Zamiri, E Rowghani, H Hejazi. 2007. Effect of a calcium soap of fatty acids on reproductive characteristics and lactation performance of fat-tailed sheep. Pak J Biol Sci 15, 2389-2395.

Gutiérrez JL. 1991. Suplementación de bovinos productores de carne en agostadero. En: Gutiérrez JL (ed). Nutrición de Rumiantes en Pastoreo. Departamento Editorial, Universidad Autónoma de Chihuahua, México, Pp 248-275.

Hawkins DE, KD Niswender, GM Oss, CL Moeller, KG Odde, HR Sawyer, GD Niswender. 1995. An increase in serum lipids increases luteal lipid content and alters the disappearance rate of progesterone in cows. J Anim Sci 73, 541-545.

Hess BW, SL Lake, EJ Scholljegerdes, TR Weston, V Nayigihugu, JDC Molle, GE Moss. 2005. Nutritional controls of beef cow reproduction. J Anim Sci 83 (Suppl E), E90-E106.

Kokta TA, AL Start, MR Papasani, JI Szasz, MV Dodson, RA Hill. 2008. Regulation of lipid accumulation in 3T3-L1 cells: insulin-independent and combined effects of fatty acids and insulin. Animal 2, 92-99.

López S, J López, W Stumpf Junior. 2007. Produção e composição do leite e eficiência alimentar de vacas da raça Jersey suplementadas com fontes lipídicas. Arch Latinoam Prod Anim 15, 1-9.

Mahecha L, J Angulo, B Salazar, M Cerón, J Gallo, CH Molina, EJ Molina, JF Suárez, JJ Lopera, M Olivera. 2008. Supplementation with bypass fat in silvopastoral systems diminishes the ratio of milk saturated/ unsaturated fatty acids. Trop Anim Health Prod 40, 209-216.

Moallem U, Y Folman, D Sklan. 2000. Effects of somatotropin and dietary calcium soaps of fatty acids in early lactation on milk production, dry matter intake, and energy balance of high-yielding dairy cows. J Dairy Sci 83, 2085-2094.

Moallem U, M Katz, A Arieli, H Lehrer. 2007. Effects of peripartum propylene glycol or fats differing in fatty acid profiles on feed intake, production, and plasma metabolites in dairy cows. J Dairy Sci 90, 3846-3856.

Morelos OS. 1988. La Vegetación: una aproximación a través de la foto interpretación en la Sierra de La Laguna de Baja California Sur. Centro de Investigaciones Biológicas de B.C.S. A.C. La Paz, B. C. S. México.

NRC, National Research Council. 1984. Nutrient Requirements of Beef Cattle. $6^{\text {th }}$ ed. National Academy Press, Washington DC, USA.

Santos JEP, TR Bilby, WW Thatcher, CR Staples, FT Silvestre. 2008. Long chain fatty acids of diet as factors influencing reproduction in cattle. Reprod Dom Anim 43 (Suppl 2), 23-30.

SAS USER'S GUIDE: Statistics. Version 1989. SAS Inst. Inc., Cary, NC, USA.

Sklan D, U Moallem, Y Folman. 1991. Effect of feeding calcium soaps of fatty acids on production and reproductive responses in high producing lactating cows. J Dairy Sci 74, 510-517.

Sklan D, R Ashkenazi, A Braun, A Devorin, K Tabori. 1992. Fatty acids, Calcium soaps of fatty acids, and cottonseed fed to high yielding cows. J Dairy Sci 75, 2463-2472.

Thomas MG, GL Williams. 1996. Metabolic hormone secretion and FSH-induced superovulatory responses of beef heifers fed dietary fat supplements containing predominantly saturated or polyunsaturated fatty acids. Theriogenology 45, 451-458.

Titgemeyer EC, CA Loëst. 2001. Amino acid nutrition: Demand and supply in forage-fed ruminants. J Anim Sci 79 (Suppl E), E180-E189.

Whitman RH. 1975. Weight changes, body condition and beef-cow reproduction. Ph.D. Dissertation, Colorado State Univ, Fort Collins, CO, USA.

Williams GL, RL Stanko. 2000. Dietary fats as reproductive nutraceuticals in beef cattle. J Anim Sci 77, 1-12.

Zachut M, A Arieli, H Lehrer, N Argov, U Moallem. 2008. Dietary unsaturated fatty acids influence preovulatory follicle characteristics in dairy cows. Reproduction 135, 683-692. 